\title{
A New Monoterpene Glucoside from Portulaca oleracea
}

\author{
Youngwan Seo, Jongheon Shin, ${ }^{*}$ Hyo Jun Cha, You-Ah Kim, \\ Jong-Woong Ahn, Burm-Jong Lec, and Dong Seok Lec ${ }^{\S}$ \\ Division of Ocean Science, Korea Marime University. Busan 606-791, Korea \\ "Natural Products Research Institute, College of Pharmacy. Seow Natonal Universilv, Seot I10-460. Korea \\ ${ }^{\ddagger}$ Department of Chemistry, Inje University, Gimhae 621-749, Korea

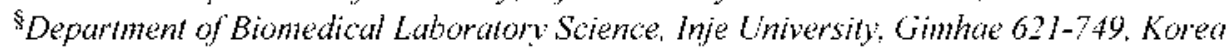 \\ Received Jily: 22, 2003
}

\begin{abstract}
Three monoterpene glucosides (1-3), including one new compound (3), have been isolated from the methanol extract of Portulaca oleracea. Structures of these compounds were determined to be (3.5)-3-O-( $\beta$-I)glucopyranosyl)-3,7-dimethylocta-1,6-dien-3-ol (1), (3S)-3-O-( $\beta$-1)-glucopyranosyl)-3,7-dimethylocta- 1,5dien-3,7-diol (2) and (3S)-3-O-( $\beta$-D)-glucopyranosyl)-3,7-dimethyl-7-hydroperoxyocta-1,5-dien-3-ol (3), respectively, by a combination of spectral analyses. Their stereochemistries were established by measurement of NOF and vicinal proton-proton coupling constants as well as comparisons of spectral data with those of previously related compounds.
\end{abstract}

Key Words : Portulaca oleracea. Portuloside B. Monoterpene glucosides, Linalool

\section{Introduction}

Portulaca oleracea Linne, known as purslane, is a succulent herb which inhabits various parts of temperate regions. It has been not only used as vegetable' but also widely used in traditional folk medicine to treat sore nipples, gastric ulcers. consillitis, and so on. ${ }^{2.3} \wedge$ number of investigators have examined extracts of $P$. oleracea on diverse bioactivities such as hepaloprotective, ${ }^{4}$ diurctic, ${ }^{4}$ anti-inflammatory, ${ }^{4.5}$ and antimicrobial ${ }^{6}$ activities as well as anti-cancer ${ }^{7}$ and antigastric ulcer. ${ }^{8}$ Therelore, it is generally conceived to contain various bioactive compounds. During the course of our search for novel metabolites from herbal medicines, we recently reported the structures of two biophenolic glycosides from the purslane. " In addition to these compounds, "I I NMR spectral analysis ol moderately polar chromatographic fractions revealed the presence of structurally different group of metabolites. Herein we report the isolation and structural determination of compounds 1-3, monoterpene glucosides.

\section{Results and Discussion}

The methanol extract of $P$. oleracea was partitioned between methylene chloride and water. The organic layer was re-partitioned between $15 \%$ aqueous $\mathrm{MeOH}$ and $n$ hexane. Purification of the aqueous $\mathrm{MeOH}$ fraction was done using $\mathrm{C} 18$ reversed-phase gravity column, followed by reversed-phase HPI.C, resulting in the isolation of monoterpene glucosides 1-3.

Compound 1 was isolated as a colorless gum which was analyzed for $\mathrm{C}_{16} \mathrm{H}_{28} \mathrm{O}_{6}$ by a combination of HRFABMS and ${ }^{13} \mathrm{C}$ NMR spectrometry. In the 'H NMR spectrum of 1 , a signal for an anomeric proton at $\delta 4.34(1 \mathrm{H}, \mathrm{d}, 7.7 \mathrm{~Hz}$ ) was correlated with a carbon signal at $\delta 99.45$ by an HMQC

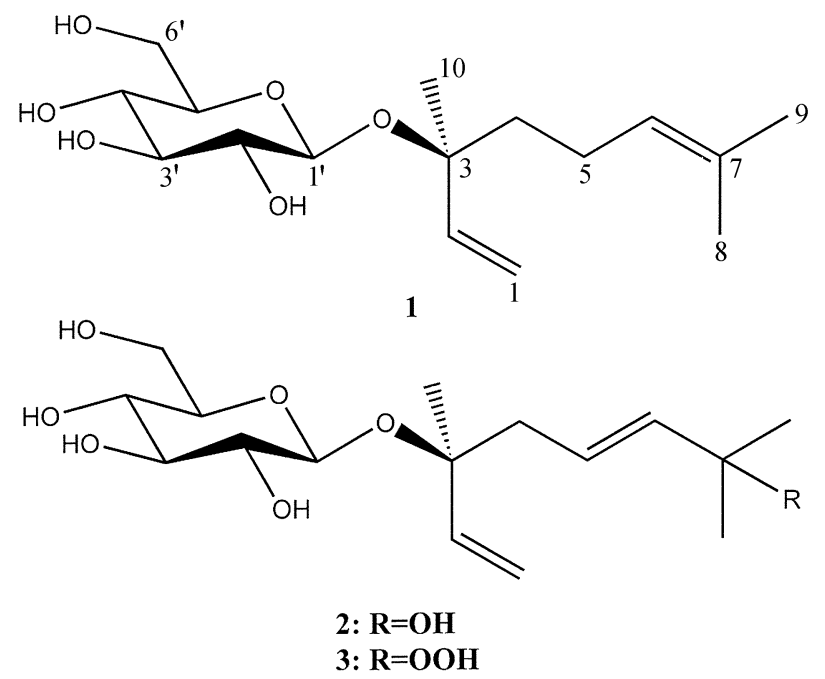

Figure 1. The Structure of compounds 1-3.

experiment, and were diagnostic for the presence of a sugar moiety, which was also supported by the presence of carbon signals in the region of $\delta 80-60$ in the ${ }^{13} \mathrm{C}$ NMR spectrum.

The overall structure of I was determined by a combination of ${ }^{1} \mathrm{H}$ COSY, TOCSY, NOESY, HSQC, and HMBC experiments. The HMBC data were particularly helpful in determining both the partial structures and connectivities among them. The presence of a terminal trisubstituted double bond was revealed by the long-ranged coupling of the olefinic proton signal $(\delta 5.08$, br $t, 7.2)$ with those of vinyl methyl protons $\left(\delta 1.59, \mathrm{br} s ; 1.66\right.$, br s) in the ${ }^{1} \mathrm{H}$ COSY experiment. Also, the ${ }^{1} \mathrm{H}$ COSY and HSQC experiments showed the presence of a monosubstituted double bond and a $\mathrm{CH}_{2} \mathrm{CH}_{2}$ unit. Determination of connectivities between these partial structures as well as an spin-isolated methyl group $(\delta 1.34,3 \mathrm{H} ; 23.20 \mathrm{q})$ and a quaternary carbon 


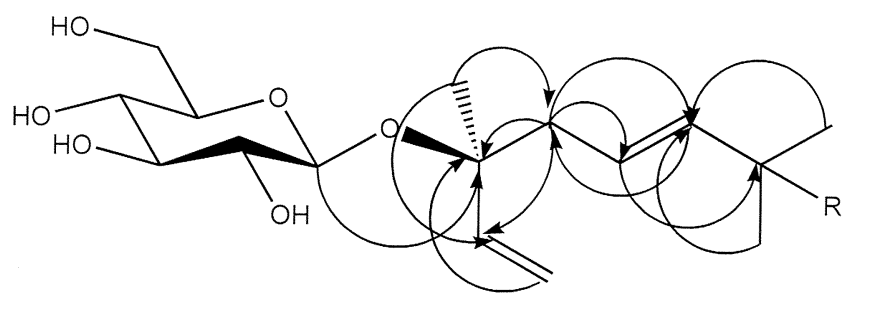

2: $\mathrm{R}=\mathrm{OH}$

3: $\mathrm{R}=\mathrm{OOH}$

Figure 2. Key IIMBC correlation of compounds 2 and 3.

$(\delta 81.37)$ signal by HMBC experiment defined the aglycon of 1 as linalool moiety. ${ }^{10}$

The planar structure of the sugar moiety was elucidated on the basis of ' $\mathrm{H}$ COSY and HMBC experiments. Its stereochemistry was determined to be $\beta$-glucopyranose by measurement of vicinal proton-proton coupling constants

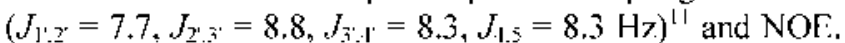
The orientations of $\mathrm{H}-\mathrm{I}^{\prime}(\delta 4.34), \mathrm{H}-3^{\prime}(\delta 3.33)$, and $\mathrm{H}-5^{\prime}(\delta$ 3.17 ) were assigned axial, axial, and axial, respectively, by the mutual NOESY correlations among them. A linkage between the aglycon and sugar moiety was established by a HMBC correlation of the $\mathrm{H}-\mathrm{l}^{\prime}$ anomeric proton of glucopyranose at $\delta 4.34$ with $\mathrm{C}-3$ of linalool part at $\delta 81.37$. With the aid of all this information, a literature survey revealed that I was identical to (3S)-3-O-( $\beta$-D-glucopyranosyl)-3,7dimethylocta-1,6-dien-3-ol (linaloyl glucoside), previously isolated from Pluchea indica and Cumila spicates ${ }^{10,11} \mathrm{Com}-$ parison of spectral data showed very good correlation with published data for this compound.

A closely related metabolite, compound 2, was isolated as a colorless gum which analyzed for $\mathrm{C}_{16} \mathrm{H}_{28} \mathrm{O}_{7}$ by HRFABMS and ${ }^{19} \mathrm{C}$ NMR analyses. Spectral data for this compound were similar to those obtained for $\mathbf{1}$. However, there are significant differences in the ${ }^{13} \mathrm{C}$ NMR spectrum. The most noticeable change is the replacement of the methylene carbon signal at $\delta 23.69$ by an oxygen-bearing quaternary carbon signal at $\delta 71.27$. Also, trisubstituted olefinic carbon signals ( $131.99 \mathrm{~s}$ and $125.55 \mathrm{~d}$ ) are changed to disubstituted olefinic ones $(142.20 \mathrm{~d}$ and $123.04 \mathrm{~d})$. Corresponding differences were found in 'H NMR spectrum in which two methylene protons at $\delta 1.58(2 \mathrm{H}, \mathrm{t}, 8.4)$ and $2.02(2 \mathrm{H}$. dt. 7.2. 8.4) merged into one methylene protons signal at $\delta 2.32$ $(2 \mathrm{H}, \mathrm{d}, 6.0)$, and new downfield signals appeared at $\delta 5.66$ $(1 \mathrm{H}, \mathrm{dt}, 15.4,6.0)$ and $5.58(1 \mathrm{H}, \mathrm{d}, 15.4)$ instead of the olefinic proton at $\delta 5.08(\mathrm{lH}$, br t. 7.2). In addition, two methyl signals at $\delta 1.59(3 \mathrm{H}, \mathrm{s})$ and $1.66(3 \mathrm{H}, \mathrm{s})$ were shifted downfield to $\delta 1.25(6 \mathrm{H}, \mathrm{s})$. On the basis of the ${ }^{1} \mathrm{H} \operatorname{COSY}$ and HSQC experiments, the locations of the double bond and hydroxyl group were assigned to C-5 and C-7, respectively, forming an en-ol functionality. This interpretation was further supported by HMBC data in which several two- and three-bond correlations were observed between the $\mathrm{C}-5$ and C-6 olefinic carbons and neighboring protons. The geometry of the double bond was assigned as $\Gamma$ by measuring a vicinal coupling constant $(15.4 \mathrm{~Hz}$.) between the olefinic protons. Thus, the structure of 2 was determined as (3S)-3-O- $(\beta-\mathrm{D}-$ glucopyranosyl)-3,7-dimethylocta-1,5-dien-3,7-diol. l.iterature survey revealed that compound 2 has not appeared as a natural product. Indeed this compound has been reported as a synthetic product of reductive cleavage of (3S)-3-O-(3', $4^{\prime}-$ diangeloyl- $\beta$-D-glucopyranosyloxy)-7-hydroperoxy-3,7-dimethylocta $-1,5$-diene. ${ }^{12}$

Another related compound 3 , designated as portuloside $B$, was obtained as a colorless gum. HRFABMS and ${ }^{1.3} \mathrm{C}$ NMR

Table 1. Carbon N.MR Assignments for Compounds $1-3^{\prime \prime}$

\begin{tabular}{|c|c|c|c|c|c|c|}
\hline \multirow{2}{*}{ no } & \multicolumn{2}{|l|}{1} & \multicolumn{2}{|l|}{2} & \multicolumn{2}{|l|}{3} \\
\hline & $\delta_{\mathrm{H}}$ & $\delta$ & $\delta_{H}$ & $\delta$ & $\delta_{\mathrm{H}}$ & $\delta$ \\
\hline \multirow[t]{2}{*}{1} & $5.2 \mathrm{l}(1 \mathrm{H} . \mathrm{dd} .17 .6 .1 .1)$ & $115.65 \mathrm{t}$ & $5.20(1 \mathrm{H} . \mathrm{dd} .17 .9 .1 .1)$ & $116.05 t$ & $5.20(\mathrm{lH} . \mathrm{dd} .17 .9 .1 .4)$ & $115.99 \mathrm{t}$ \\
\hline & $5.19(1 \mathrm{H} . \mathrm{dd} .10 .8 .1 .1)$ & & $5.19(1 \mathrm{H} . \mathrm{dd} .10 .7 .1 .1)$ & & $5.19(\mathrm{IH} . \mathrm{dd} .10 .7 .1 .4)$ & \\
\hline 2 & $5.91(1 \mathrm{H} . \mathrm{dd} .17 .6 .10 .8)$ & $144.31 \mathrm{~d}$ & $5.91(1 \mathrm{H} . \mathrm{dd} .17 .9 .10 .7)$ & $143.84 \mathrm{~d}$ & $5.92(1 \mathrm{H}$. dd. 17.9 .10 .7$)$ & $143.91 \mathrm{~d}$ \\
\hline 3 & & $81.37 s$ & & $81.32 \mathrm{~s}$ & & $81.37 \mathrm{~s}$ \\
\hline 4 & $1.58(2 \mathrm{H} . \mathrm{t} .8 .4)^{\mathrm{h}}$ & $42.66 \mathrm{t}$ & $2.32(2 \mathrm{H}$. d. 6.0$)$ & $45.34 \mathrm{t}$ & $2.35(2 \mathrm{H}$. d. 6.2$)$ & $45.50 \mathrm{t}$ \\
\hline 5 & $2.02(2 \mathrm{H} . \mathrm{dt} .7 .2 .8 .4)$ & $23.69 \mathrm{t}$ & $5.66(1 \mathrm{H}$. dt. 15.4 .6 .0$)$ & $123.04 \mathrm{~d}$ & $5.67(1 \mathrm{H} . \mathrm{dt} .16 .0 .6 .2)$ & $126.68 \mathrm{~d}$ \\
\hline 6 & $5.08(111$, br $(.7 .2)$ & $125.55 \mathrm{~d}$ & $5.58(11, d, 15.4)$ & $142.20 \mathrm{~d}$ & $5.59(1 \mathrm{H} . \mathrm{d} .16 .0)$ & $138.63 d$ \\
\hline 7 & & $131.99 s$ & & $71.27 \mathrm{~s}$ & & $82.50 \mathrm{~s}$ \\
\hline 8 & $1.59(311, s)$ & 16.119 & $1.25\left(31 \mathrm{I}_{n} \mathrm{~s}\right)$ & $29.97 \mathrm{G}$ & $1.27(3 \mathrm{H}, \mathrm{s})$ & $23.41 \mathrm{q}$ \\
\hline 9 & $1.66(311 . s)$ & $25.92 \mathrm{q}$ & $1.25(311.5)$ & $29.97 \mathrm{q}$ & $1.27(3 \mathrm{H} . \mathrm{s})$ & $24.98^{\circ} \mathrm{q}$ \\
\hline 10 & $1.34(31 \mathrm{~s} . \mathrm{s})$ & $23.20 \mathrm{q}$ & $1.34(31 . . s)$ & $23.32 \mathrm{q}$ & $1.34(3 \mathrm{H} . \mathrm{s})$ & $24.93^{\circ} \mathrm{q}$ \\
\hline$l^{\prime}$ & 4.34 (111.d. 7.7$)$ & $99.45 \mathrm{~d}$ & $4.36(11 . \mathrm{d} .7 .7)$ & $99.41 \mathrm{~d}$ & 4.36 (1H. d. 7.7$)$ & $99.47 \mathrm{~d}$ \\
\hline $2^{\prime}$ & $3.15(1 \mathrm{H} . \text { dd. } 8.3 \cdot 7.7)^{b}$ & $75.15 \mathrm{~d}$ & $3.15(1 \mathrm{H} . \mathrm{dd} .8 .8 \cdot 7.7)^{h}$ & $75.10 \mathrm{~d}$ & $3.15(1 \mathrm{H} . \mathrm{dd} \cdot 8.7 .7 .7)^{h}$ & $75.13 \mathrm{~d}$ \\
\hline 3 & $3.33(1 \mathrm{H} . \text { dd. } 8.8 .8 .3)^{b}$ & $78.28 \mathrm{~d}$ & $3.33(1 \mathrm{H} . \mathrm{dd} .8 .8 .8 .3)^{h}$ & $78.08 \mathrm{~d}$ & $3.33(1 \mathrm{H} . \mathrm{dd} .8 .7 .8 .0)^{h}$ & $78.15 \mathrm{~d}$ \\
\hline $4^{\prime}$ & $3.27(1 \mathrm{H}$. dd. 8.3 .8 .3$)$ & $71.69 \mathrm{~d}$ & $3.27(1 \mathrm{H}$. dd. 8.3. 8.3) & $71.64 \mathrm{~d}$ & $3.26(1 \mathrm{H}$. dd. 8.0 .8 .0$)$ & $71.68 \mathrm{~d}$ \\
\hline $5^{\prime}$ & $3.17(1 \mathrm{H} . \mathrm{IH} . \mathrm{m})$ & $77.55 \mathrm{~d}$ & $3.16(1 \mathrm{H} . \mathrm{IH} . \mathrm{m})$ & $77.54 \mathrm{~d}$ & $3.17(1 \mathrm{H} .1 \mathrm{H} . \mathrm{m})$ & $77.60 \mathrm{~d}$ \\
\hline \multirow[t]{2}{*}{$6^{\prime}$} & $3.79(1 \mathrm{H} . \mathrm{dd} .11 .8 .2 .5)$ & $62.79 \mathrm{t}$ & $3.80(1 \mathrm{H} . \mathrm{dd} .12 .1 .2 .5)$ & $62.71 \mathrm{t}$ & $3.80(\mathrm{IH} . \mathrm{dd} . \mathrm{II} .8 .2 .2)$ & $62.76 \mathrm{t}$ \\
\hline & $3.62(1 \mathrm{H} . \mathrm{dd} .11 .8 .5 .5)$ & & $3.62(1 \mathrm{H} . \mathrm{dd} .12 .1 .5 .7)$ & & $3.62(\mathrm{IH}$. dd. II.8.5.5) & \\
\hline
\end{tabular}

"II and ${ }^{15} \mathrm{C}$ NMR spectra were recorded in CD,OD solution at 300 and 75 . $\mathrm{Ml}$ I7., respectively. Assignments were based upon 'II COSY, IISOC. and IIMBC experiments. "Coupling constants were measured by $1 D$ TOCSY experiment. "Txchangeable. 
spectral data of this compound showed the molecular formula $\mathrm{C}_{16} \mathrm{H}_{28} \mathrm{O}_{8}$. ${ }^{1} \mathrm{H}$ NMR spectral data for this compound were almost the same as those of 2 . The only significant difference in the ${ }^{15} \mathrm{C}$ NMR spectrum was the downfield shift of a carbon signal from $\delta 71.27$ for 2 to $\delta 82.50$. Its neighboring carbon signals also exhibited considerable changes contpared with 2 ( $\Delta \delta 3-5 \mathrm{ppm}$ for $\mathrm{C}-5,-6 .-8$. and -9). These changes were accommodated by replacement of the hydroxy group with hydroperoxy one which naturally influences stronger electron-withdrawing effect than the fonmer group. This interpretation was also supported by HMBC correlations between the carbon signal at $\delta 82.50$ and its neighboring protons ( $\mathrm{H}-5 .-8$, and -9). In addition. NMR spetral data for 3 were well-matched with those for 3'. 4'-diangeloate of 3, previously isolated from Aster scaber. ${ }^{12}$ Thus. the structure of portuloside B was confirmed as (3S)3-O-( $\beta$-D-glucopyranosyl)-3,7-dimethyl-7-hydroperoxyocta1,5-dien-3-ol.

\section{Experimental Section}

General Experimental Procedures. Optical rotations were obtained on a Jasco digital polarineter. NMR spectra were recorded in $\mathrm{CD}_{3} \mathrm{OD}$ on Varian Mercury 300 spectrometers using standard pulse sequence progranis. Proton and carbon NMR spectra were measured at 300 and $75 \mathrm{MHz}$. respectively. Chemical shifts were recorded with respect to $\mathrm{CD}_{3} \mathrm{OD}$ as an internal standard. Mass spectral data were obtained at the Korean Basic Science Institute. Taejeon. Korea. High performance liquid chromatography (HPLC) was performed with a Dionex P580 with a Varian 350 RI detector. All solvents used were spectral grade or were distilled from glass prior to use.

Extraction and Isolation. Dried samples $(0.5 \mathrm{~kg})$ of $P$. olerwer were ground to a powder and repeatedly extracted for 2 days with $\mathrm{MeOH}(3 \mathrm{~L})$. The combined crude extracts $(29.8 \mathrm{~g}$ ) were evaporated to dryness and partitioned between $\mathrm{CH}_{2} \mathrm{Cl}_{2}$ and water. The organic layer was further partitioned between $15 \%$ aqueous $\mathrm{MeOH}$ and $n$-hexane to give a $15 \%$ aqueous $\mathrm{MeOH}$ fraction (12.1 g), which was subjected to $\mathrm{C}_{1 \mathrm{~s}}$ reversed-phase vacuum flash chromatography eluting with stepwise gradient mixtures of $\mathrm{MeOH}$ and water $(50 \%$. $40 \%, 30 \%, 20 \%, 10 \%$ aqueous $\mathrm{MeOH}$, and $100 \% \mathrm{MeOH}$ ). The fraction $(4.7 \mathrm{~g}$ ) eluted with $30 \%$ aqueous $\mathrm{MeOH}$ was dried and the residue was separated with $\mathrm{Si}$ gel chromatography using a gradient solvent system of $\mathrm{CHCl}_{3}-\mathrm{MeOH}$. Further purification of $20 \% \mathrm{MeOH}$ in chloroform fraction $(0.9 \mathrm{~g})$ by PTLC on a Si gel with $\mathrm{MeOH} / \mathrm{CHCl}_{3}(1: 20)$ as a solvent șystem followed by semi-preparative $\mathrm{C}_{18}$ HPLC (YMC ODS-A columu. $1 \mathrm{~cm} \times 25 \mathrm{~cm} .90 \%$ aqueous $\mathrm{MeOH}$ ) gave compounds 1 (10.8 mg). 2 (3.2 $\mathrm{mg})$. and 3 (2.1 mg).
(3S)-3-0-( $\beta$-D-Glucopyranosyl)-3,7-dimethylocta-1,5dien-3-ol (1): $[\alpha]_{D}^{25}-12.5^{\circ}$ (c l.1. $\mathrm{CHCl}_{2}$ ) (lit. ${ }^{1 \mathrm{li}}[\alpha]_{\mathrm{D}}^{24}-23.4$ ); FABMS $m=339.1782(\mathrm{M}+\mathrm{Na})^{-}$(calcd for $\mathrm{C}_{16} \mathrm{H}_{28} \mathrm{O}_{8} \mathrm{Na}$, 339.1784): HMBC correlations $\mathrm{H}-1 / \mathrm{C}-2$. C-3; H-4/C-2. C-3, $\mathrm{C}-5 ; \mathrm{H}-5 / \mathrm{C}-6, \mathrm{C}-7: \mathrm{H}-8 / \mathrm{C}-6, \mathrm{C}-7, \mathrm{C}-9 ; \mathrm{H}-9 / \mathrm{C}-6, \mathrm{C}-7 . \mathrm{C}-8$; $\mathrm{H}-\mathrm{I} 0 / \mathrm{C}-2, \mathrm{C}-3 . \mathrm{C}-5: \mathrm{H}-\mathrm{I}^{1} / \mathrm{C}-3 ;{ }^{1} \mathrm{H}$ and ${ }^{13} \mathrm{C}$ NMR spectral data. see Table 1 .

(3S)-3-0-( $\beta$-D-Glucopyranosyl)-3,7-dimethylocta-1,5dien-3,7-rliol (2): a colorless gum; $[\alpha]_{\mathrm{D}}^{25}-12.4^{\circ}$ (c 0.3 , $\mathrm{MeOH})$ : HRFABMS $m: z 355.1731(\mathrm{M}+\mathrm{Na})^{-}$(calcd for $\mathrm{C}_{16} \mathrm{H}_{28} \mathrm{O}_{7} \mathrm{Na} .355 .1733$ ); HMBC correlations $\mathrm{H}-1 / \mathrm{C}-2$. C-3; $\mathrm{H}-4 / \mathrm{C}-2$. C-3. C-5: H-5/C-7; H-6/C-4; H-8/C-6. C.7; H-9/C6. $\mathrm{C}-7 ; \mathrm{H}-10 / \mathrm{C}-2 . \mathrm{C}-3, \mathrm{C}-4: \mathrm{H}-1^{1 / C} \mathrm{C}-3:{ }^{1} \mathrm{H}$ and ${ }^{13} \mathrm{C}$ NMR spectral data, see Table 1 .

Portuloside B, (3S)-3-0-( $\beta$-D-Glucopyranosyl)-3,7-dimethyl-7-hydroperoxyocta-1,5-dien-3-ol (3): a colorless gum: $[\alpha]_{\mathrm{D}}^{25^{\circ}}-3.1^{\circ}$ (c 0.2, MeOH): HRFABMS $m z 371.1681$ $(\mathrm{M}+\mathrm{Na})^{-}$(calcd for $\mathrm{C}_{16} \mathrm{H}_{28} \mathrm{O}_{8} \mathrm{Na}$. 371.1682): $\mathrm{HMBC}$ correlations $\mathrm{H}-1 / \mathrm{C}-2, \mathrm{C}-3 ; \mathrm{H}-4 / \mathrm{C}-2, \mathrm{C}-3, \mathrm{C}-5 ; \mathrm{H}-5 / \mathrm{C}-7 ; \mathrm{H}-6 / \mathrm{C}-4$; $\mathrm{H}-8 / \mathrm{C}-6 . \mathrm{C}-7: \mathrm{H}-9 / \mathrm{C}-6, \mathrm{C}-7 ; \mathrm{H}-10 / \mathrm{C}-2, \mathrm{C}-3 . \mathrm{C}-4 ; \mathrm{H}-1 / \mathrm{C}-3 ;$ ${ }^{1} \mathrm{H}$ and ${ }^{13} \mathrm{C}$ NMR spectral data. see Table $\mathrm{I}$.

Acknowledgement. This research was supported by a grant (\#RI2-2001-006304-0) of the Korea Science and Engineering Foundation (KOSEF) tlurough the Biolnealth Products Research Center (BPRC), Inje University. Republic of Korea. We thank Mr. Yang-Ho Park for providing specimens of $P$. oleracea. Mass spectral data were kindly provided by Korea Basic Science Institute.

\section{References}

1. Mohamed. A. I.: Hussein. A. S. Plan Foods for Human Futrition 1994. $+5,1$

2. Choi. K.-P.: Jung. S.-W.: Kim. E.-J.- Ham, S.-S. Journal of the East Asian of Dietary Life 1997. 7, 527 .

3. Ghazantar. S. A. Handbook of Arabian Hedicind Plants: CRC Press: Boca Raton. FL. 1994: p 176.

4. Liml. J. P.: Sull. E. S. Konean J. Medicinal Chop Sei. 2000. 8. 189.

5. Chan. K: Islam. M: Kamil. M: Radhakrishnan, R.: Zaharia, M. N. M.: Habilullah. M.: Attas, A. Jonnal of Elmopharntacology 2000. 73,445

6. Lim. M.-K.: Kim. M. Korean J. Soc. Food. Cookery Sci. 2001. 17. 565.

7. Yoon1. T. W.: Hamn. S. S.: Tut1. H. S. LS.S. Pat. 5869060.1999.

8. Islam. M. W.: Zaharia. M. N. M.: Radhakrishnan, R.: Liu. X. M: Chen. H. B.: Chan. K: Al-Attas, A. J. Phamt. Phamacol 2000. 52.258 .

9. Seo. Y: Shitr. J.: Lee. B.-J.: Lee D. S. J. Kor?an Chem. Soc. 2003. 47. 1 .

10. Uchiyama. T.: Miyase. T: Ueno. A.: Usmanghanil. K. Plntochentisny 1989. 28, 3369

11. Manns. D. Phwochentistry 1995.39,1115

12. Jung. C. M: Kwon, H. C.: Seo. J. J.; Ohizumi, Y: Matsunaga, K. Saito. S.: Lee. K. R. Chem. Phom. Bull. 2001. 19.912. 